\title{
How should ecohazard of micropollutants in wastewater be gauged? Using bioassays to profile alternative tertiary treatments
}

\author{
Matteo Papa ${ }^{1,2^{+*}}$, Lidia Paredes ${ }^{1^{*}}$, Donatella Feretti ${ }^{3,6}$, Gaia Viola ${ }^{3}$, Giovanna Mazzoleni ${ }^{4,6}$, \\ Nathalie Steimberg ${ }^{4,6}$, Roberta Pedrazzani ${ }^{5,6}$, Juan Lema $^{1}$, Francisco Omil ${ }^{1}$, Marta Carballa ${ }^{1}$ \\ ${ }^{1}$ Department of Chemical Engineering, Institute of Technology, Universidade de Santiago de Compostela, Rua Lope Comez de Marzoa, E-15782 \\ Santiago de Compostela, Spain \\ ${ }^{2}$ Department of Civil, Environmental, Architectural Engineering and Mathematics, University of Brescia, via Branze 43, I-25123 Brescia, Italy \\ ${ }^{3}$ Department of Medical and Surgical Specialties, Radiological Sciences and Public Health, University of Brescia, Viale Europa 11, I-25123 Brescia, Italy \\ ${ }^{4}$ Department of Clinical \& Experimental Sciences, University of Brescia, viale Europa 11, I-25123 Brescia, Italy \\ ${ }^{5}$ Department of Mechanical and Industrial Engineering, University of Brescia, via Branze 38, 1-25123 Brescia, Italy \\ ${ }^{6}$ Brescia University Research Center "Integrated Models for Prevention and Protection in Environmental and Occupational Health" (MISTRAL), Italy \\ *These authors contributed equally to this work
}

\begin{abstract}
The research on emerging pollutants in wastewater has become a worldwide issue of increasing environmental concern, especially considering the growing interest in wastewater reuse. However, the latter implies additional post-treatment after the conventional activated sludge processes, in order to produce a safer effluent. Our work aimed at determining the efficiency of reducing the toxicity associated with organic micropollutants (OMPs) in secondary wastewater effluents, using 3 different post-treatment technologies (granular activated carbon (GAC), sand biofiltration and UV irradiation): in particular, target chemical analysis of the OMPs most commonly founded in wastewater was coupled with effect-based assays (estrogenicity and mutagenicity). While chemical analysis assessed satisfactory performances for all 3 technologies in the abatement of selected OMPs, biological assays evidenced another perspective: both GAC and sand biofilters were significantly able to make the estrogenic load plummet; however, the UV system was ineffective in estrogenicity abatement, and its effluent exhibited also a slight mutagenicity, likely due to photo-transformation by-products. These results indicate that a synergistic combination of chemical analysis and biological assays can drive to a proper gauging of post-treatment technologies, taking into account not only the removal of OMPs, but also their overall toxicity.
\end{abstract}

Keywords: Estrogenicity, Granular activated carbon, Mutagenicity, Organic micropollutants, Sand filtration, UV irradiation

\section{Introduction}

The research on organic micropollutants (OMPs) in wastewater has become a worldwide issue of increasing environmental concern, especially considering the growing interest in the reuse of treated wastewater. Thus, the application of post-treatment technologies such as biofiltration systems, UV irradiation or advanced oxidation processes is considered a promising solution to adequate the quality of secondary effluents in view of an eventual reclamation [1].

Despite the large number of studies investigating the fate and removal of various individual and specific groups of OMPs after post-treatments, so far little is known whether these processes affect the toxic activity of OMPs themselves and, consequently, of the effluents. This underdevelopment is mainly due to a lack of standardized protocols for the selection and the execution of biological assays, especially for specific modes of toxic action [2]. Another weakness is related to the use of techniques neither $i$ ) specific enough (in reaching the target endpoints) nor ii) adequately sensible. As the first issue is concerned, for instance Vibrio fischeri is frequently employed, but it is only able to detect the baseline toxicity [3]; for the second, the determination of estrogenic activity by means of traditional techniques (e.g. yeast estrogenic screen) does not allow a satisfactory degree of sensibility and robustness, while the application of human breast cancer MCF-7 cells for estro-

Received March 29, 2020 Accepted July 20, 2020

${ }^{\dagger}$ Corresponding author

Email: matteo.papa82@gmail.com

Tel: +61 755527836 Fax: +61 755527836

ORCID: 0000-0003-4872-3411

Copyright (C) 2021 Korean Society of Environmental Engineers 
genicity detection ensures accurate and reproducible results thanks to its high concentration of estrogenic receptors.

Thus, the development of sensible and specific assays represents a valuable strength when profiling tertiary treatments $[4,5]$. Indeed, effect-based monitoring approaches are required to gain information on the cumulative effects of complex mixtures characterizing wastewater [6]: the detection of OMPs alone is not sufficient to evaluate the ecohazard of treated effluent and, ultimately, the negative effects on aquatic organisms [2, 7, 8].

The objective of this work was to determine the efficiency of three post-treatment technologies (granular activated carbon (GAC) biofiltration, sand biofiltration and UV irradiation) for the mitigation of estrogenic and mutagenic activities (measured by means of MCF-7 cells and Ames test, respectively), therefore expanding the information obtained from the OMPs chemical analysis alone.

\section{Experimental Section}

\subsection{Reactors}

\subsubsection{Biofiltration systems}

Laboratory scale biofilters were used, consisting of two columns of methacrylate, each with $3 \mathrm{~L}$ of sand and granular activated carbon as filtering material, respectively. Both biofilters were fed with a secondary effluent (main characteristics: $\mathrm{pH}$ : 7.5, chemical oxygen demand (COD): $79 \mathrm{mg} / \mathrm{L}$, ammonium $\left(\mathrm{N}^{-\mathrm{NH}_{4}}\right): 0.9 \mathrm{mg} / \mathrm{L}$ and nitrate $\left.\left(\mathrm{N}^{-} \mathrm{NO}_{3}\right): 5.9 \mathrm{mg} / \mathrm{L}\right)$ that was spiked with selected OMPs (full details in Table S1 of Supplementary Materials). The detailed description of biofilters operation can be found in [9]: among a wider spectrum of operating conditions tested against OMPs removal, the effect-based assays were carried out under an empty bed contact time (EBCT) of $4.8 \mathrm{~h}$. During this period, two sampling campaigns were carried out for quantifying target OMPs and, on the second campaign, bioassays were executed on triplicate and duplicate for estrogenicity and mutagenicity, respectively.

\subsubsection{UV system}

The UV lab reactor was a $1 \mathrm{~L}$ cylindrical glass contactor provided with a low-pressure lamp (TNN 15/32, nominal power $15 \mathrm{~W}$ ) emitting monochromatic radiation at $254 \mathrm{~nm}$. Similarly to biofilters, a secondary effluent (main characteristics: pH: 7.3, COD: $24 \mathrm{mg} / \mathrm{L}$, $\mathrm{N}-\mathrm{NH}_{4}: 1.3 \mathrm{mg} / \mathrm{L}, \mathrm{NO}_{3}: 3.6 \mathrm{mg} / \mathrm{L}$ and turbidity: $4.7 \mathrm{NTU}$ ) spiked with selected OMPs was used (full details in Table S1 of Supplementary Materials). The detailed description of UV reactor operation can be found in [10]: again, among a wider spectrum of operating conditions where OMPs were detected, the effect-based assays were carried out under an UV dose of $1,200 \mathrm{~mJ} / \mathrm{cm}^{2}$. For this experiment, OMPs were determined on duplicate whereas bioassays were executed on triplicate for estrogenicity and on duplicate for mutagenicity.

It is worth to note that, for both reactors, the treatment conditions we decided to test are stronger than those usually applied in full-scale systems: especially the UV dose, usually in the range of $50-200 \mathrm{~mJ} / \mathrm{cm}^{2}$ [11]. However, these conditions were properly selected to ensure effectiveness towards not only the parent OMPs, but also their transformation products, which play a key role in the effect-based assays.

\subsection{Organic Micropollutants}

Several OMPs commonly found in secondary effluents were targeted in this work: three pain killers (ibuprofen, naproxen and diclofenac), four antibiotics (erythromycin, roxithromycin, sulfamethoxazole and trimethoprim), three psychoactive drugs (fluoxetine, carbamazepine and diazepam), three musk fragrances (galaxolide, tonalide and celestolide), three estrogens (estrone, 17 $\beta$-estradiol and $17 \alpha$ -ethinylestradiol) and four endocrine disruptor compounds - EDCs (4-octylphenol, 4-nonylphenol, bisphenol $\mathrm{A}$ and triclosan). Compound abbreviations are reported in the caption of Fig. 1.

For their determination, $250 \mathrm{~mL}$ influent and effluent samples were prefiltered ( $0.70 \mu \mathrm{m}$, Millipore) and pre-concentrated by solid phase extraction (SPE) prior to analysis with Gas Chromatography Mass Spectrometry - GC/MS (pain killers, musk fragrances and EDCs) and Liquid Chromatography Tandem Mass Spectrometry - LC/MS/MS (antibiotics, estrogens and psychoactive drugs). Further details on the limits of detection, quantification and recoveries of analytical methods are described in [9] and [10].

It is worth noting that, as we spiked samples by adding three estrogens in concentration of $1 \mu \mathrm{g} / \mathrm{L}$ (100 - 1,000 times the usual concentration in wastewater), thus E1, E2 and EE2 were by far the major estrogenic active substances, due to a relative estrogenic potency 100 - 1,000 times higher than the other spiked EDCs [12].

\subsection{Effect-Based Assays}

The same SPE as for chemical analyses was executed, and extracts dried under nitrogen flow and resuspended in $1 \mathrm{~mL}$ dimethyl sulfoxide (DMSO), leading thus to an initial enrichment factor of $250 \mathrm{~mL}_{\text {wastewater }} / \mathrm{mL}_{\text {extract }}$.

\subsubsection{Estrogenic activity}

Human breast cancer cell line MCF-7 was selected to measure the estrogenic activity. MCF-7 stably transfected with the ERE-tK-LUC construct (kindly supplied by Mikko Unkila, Hormos Medical Ltd, Turku, Finland) was maintained in DMEM (Modified Dulbecco's Medium, Milan, Italy), supplemented with 10\% fetal bovine serum, at $37^{\circ} \mathrm{C}$ and $5 \% \mathrm{CO}_{2}$. Cells were plated at a density of $2.5 \cdot 10^{5}$ cells $/ \mathrm{cm}^{2}$ in several plates containing $1 \mathrm{~mL}$ of culture medium (phenol red-free DMEM and 5\% charcoal-stripped serum). $24 \mathrm{~h}$ later, $1 \mu \mathrm{L}$ of each DMSO extract (therefore, with a dilution factor of 1,000) was added by triplicate and dishes were kept at $37^{\circ} \mathrm{C}$ for $24 \mathrm{~h}$. As controls without extracts, one cell-plate was supplemented with DMSO solvent, another with ethanol and a last one only with cells. After incubation, cells were harvested and lysed in passive lysis buffer (Promega, Italy). Lysate was spun for $15 \mathrm{~s}$ at $12,000 \mathrm{~g}$ and supernatant submitted to luciferase activity quantification (Luciferase Assay System, Promega, Italy), by means of a luminometer (GloMAx, Promega, Italy) over $10 \mathrm{~s}$, and expressed as RLU (relative light units) normalized towards protein content (Bradford assay, Biorad, Italy). The latter value was then expressed as estradiol equivalent concentration (ng-EEQ/Lbioassay), based on the calibration curve, and ultimately as ng-EEQ/ $\mathrm{L}_{\text {sample }}$ using the relative enrichment factor (REF). REF represents the combination of the initial enrichment factor and the dilution factor in the bioassay 


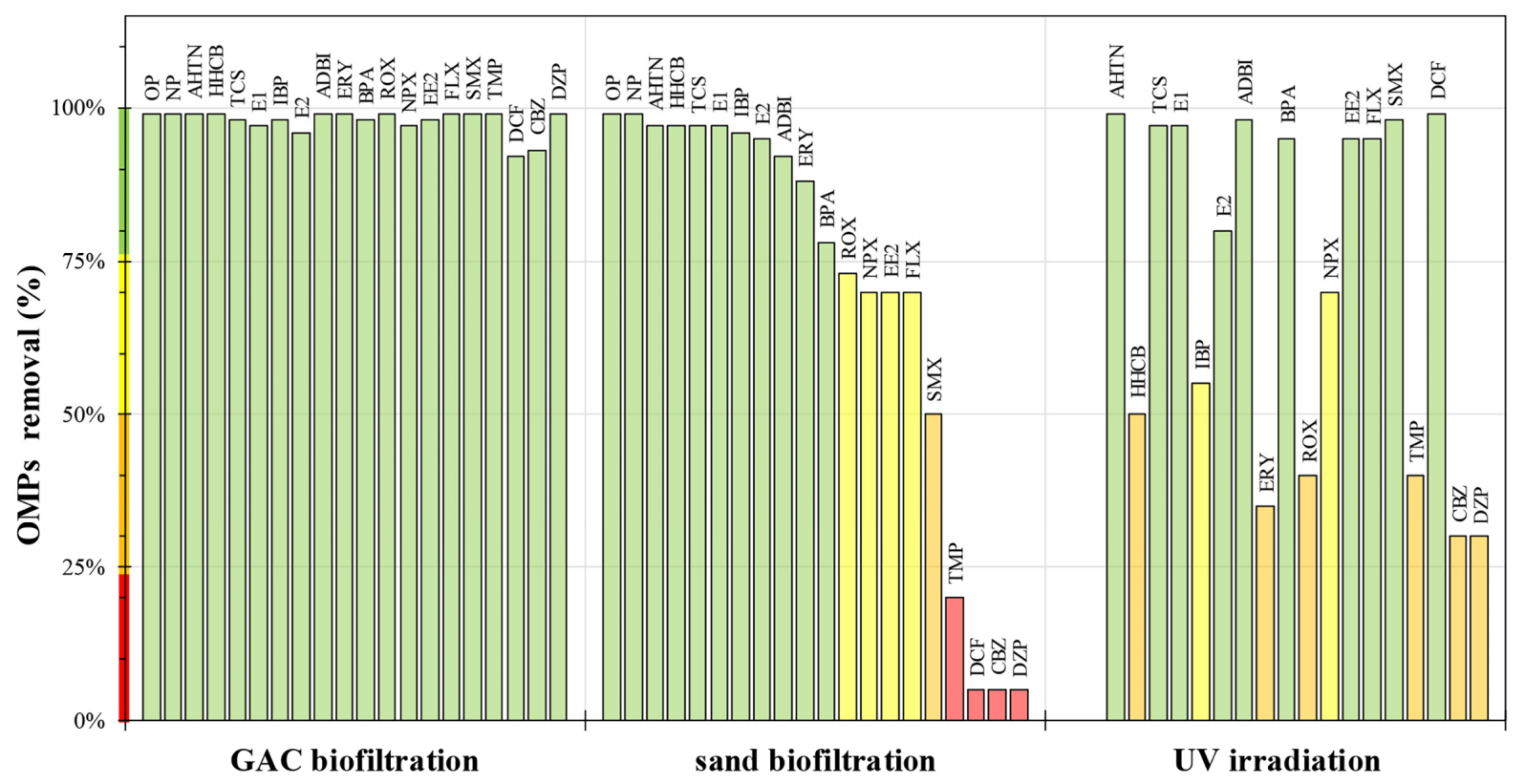

Fig. 1. Average OMPs removal efficiencies in biofilters and UV. Compounds are listed according to their behavior in sand biofilter and labelled as follows: 4-octylphenol (OP), 4-nonylphenol (NP), tonalide (AHTN), galaxolide (HHCB), triclosan (TCS), estrone (E1), ibuprofen (IBP), 17ß-estradiol (E2), celestolide (ADBI), erythromycin (ERY), bisphenol A (BPA), roxithromycin (ROX), naproxen (NPX), 17a-ethinylestradiol (EE2), fluoxetine (FLX), sulfamethoxazole (SMX), trimethoprim (TMP), diclofenac (DCF), carbamazepine (CBZ), diazepam (DZP). The colors indicate the level of abatement: green $=$ high $(>75 \%)$; yellow $=$ mild $(50 \%$ to $75 \%)$; orange $=$ low $(25 \%$ to $50 \%)$; red $=$ absent $(<$ $25 \%$ ) (for interpretation of the references to color in this figure legend, the reader is referred to the web version of this article).

plates, as explained by [13]: therefore, REF was equal to 0.25 $\mathrm{mL}_{\text {wastewater }} / \mathrm{mL}_{\text {bioassay. }}$.

For the calibration curve, reference estrogen (E2 dissolved in ethanol) was employed, at concentrations corresponding to physiological/sub-physiological doses, i.e. from $10^{-15}$ to $10^{-8} \mathrm{M}$. The resulting curve (sigmoïdal function) was fitted using Graphpad Prism 6.0 software (GraphPad Software, Inc., USA). Details of cell response to the reference estrogen, together with the calibration curve, are reported in Fig. S1 and Table S2 of Supplementary Materials.

\subsubsection{Mutagenic activity}

Mutagenicity was assessed using Ames test, which is able to evidence point mutations in bacteria; specifically, we used the TA98 strain of Salmonella typhimurium (able to detect frameshift mutagens) according to [14]. Bacteria were exposed to increasing doses of DMSO extracts: 1-, 5-, 10-, 25- and 50-mL of wastewater equivalents per plate. Positive (10 $\mu \mathrm{g} /$ plate of 2-nitrofluorene) and negative (DMSO solvent) controls were executed.

Data were expressed as mutagenicity ratio (MR), dividing the revertants/plate by the spontaneous mutation rate derived from the negative control. Results were considered positive if MR was higher than 2 (a response at least twice the negative control).

\section{Results and Discussion}

As aforementioned, we spiked target OMPs: therefore, raw values obtained from bioassays were not discussed themselves, but rather used to calculate and compare removal efficiencies, as hereinafter discussed. Raw data are however reported in Supplementary Materials (Figure S2 for estrogenic and Table S3 for mutagenic activity).

Bioassays were the main focus of this paper, while two previous papers ([9] and [10] that act as accompanying papers) were entirely devoted to the chemical analysis results, specifically explaining the behaviour of OMPs in the three studied post-treatment systems and their different removal mechanisms.

\subsection{Biofiltration Systems}

First of all, we analyzed chemical data as background information, to see what we should expect from the effect-based assays. Fig. 1 summarizes the removal efficiencies obtained for targeted OMPs: for a quicker understanding, removals were grouped into four categories with appropriate color coding.

It is clear how GAC biofilter exhibited a great potential for OMPs removal: indeed, only the green category (high removal) is represented in Fig. 1, with efficiencies always above 90\% for all targeted micropollutants. On the contrary, all the 4 categories are displayed in the case of sand biofilter, where OMPs elimination was strongly related to their biodegradability: for instance, unsatisfactory abatement characterized the most recalcitrant/hardy biodegradable compounds (such as diclofenac, carbamazepine and diazepam); the same compounds, instead, were strongly removed in GAC thanks to the adsorption on activated carbon. Further details of the removal mechanisms in [9]. 


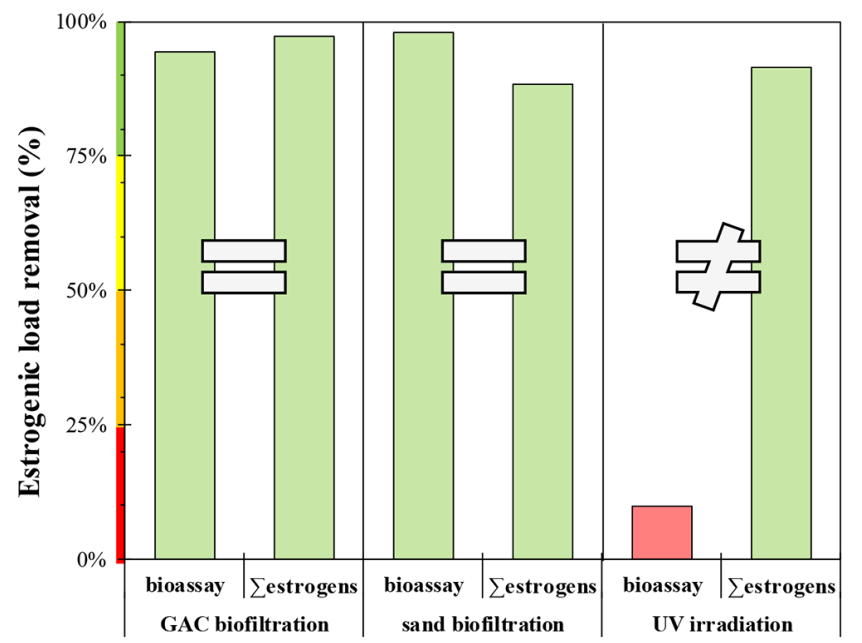

Fig. 2. Estrogenic bioassay VS chemical analysis. Average removal of estrogenic activity in biofilters and UV (bioassay response), and comparison with chemical analysis (i.e., estrogens removal). The colors indicate the level of abatement: green $=$ high $(>75 \%)$; yellow $=$ mild $(50 \%$ to $75 \%)$; orange $=$ low $(25 \%$ to $50 \%)$; red $=$ absent $(<25 \%)$ (for interpretation of the references to color in this figure legend, the reader is referred to the web version of this article).

Then, we examined the bioassays outcomes for the effect-based analysis. Fig. 2 reports the removal of estrogenic activity, calculated on the basis of estradiol equivalent concentrations (ng-EEQ/L, reported in Fig. S2 of Supplementary Materials). Both GAC and sand biofilters reached a strong abatement of estrogenicity (higher than 90\%), in agreement with the outcomes of other researches ([15] among others).

To link the removal of the most critical pollutants exhibiting estrogenic activity (i.e., the 3 estrogens) with the reduced estrogenicity, we plotted in Fig. 2 both the information (chemical and biological), and we recorded the accordance of their outcome: indeed, estrogenicity abatement (> 90\%) was strongly in agreement with estrogens removal, that also topped 90\% (except EE2 in sand biofiltration, however removed at a $70 \%$ extent). This outcome is a confirmation that no estrogenically active by-products were formed during biofiltration, as already postulated by other authors [16]: by using an approach only based on chemical analysis, it would not have been possible to get such a holistic answer, as biotransformation products would not have been taken into account.

On the other hand, mutagenic activity was not observed, neither in the influent nor in the effluent of the biofilters, since the mutagenicity ratio was always below the threshold of 2 (Fig. 3, while the raw values of revertants/plate are reported in Table S3 of Supplementary Materials). This is explained by the fact that spiked OMPs are not recognized as mutagenic compounds, and GAC and sand biofiltration do not generate transformation products able to induce frameshift mutations [16].

Notwithstanding, the lack of transformation products after biofilters is not tout court, but only related to those compounds responsible for monitored biological activities (estrogenicity and mutagenicity): indeed, the formation of transformation products in/during biofiltration is evident elsewhere [17] for other OMPs.

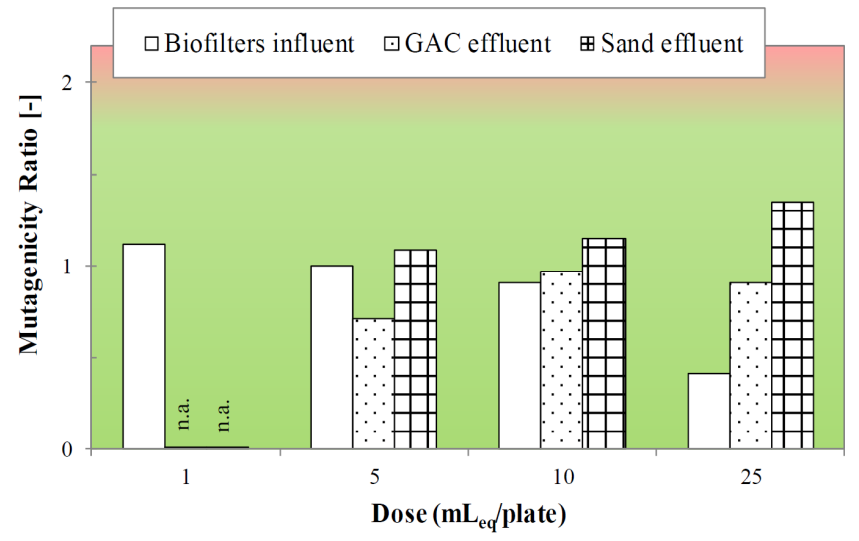

Fig. 3. Average Mutagenicity Ratio obtained using Ames test, for GAC and sand biofilters (n.a. = not available). Background color indicates a negative (green $=M R<2$ ) or positive $($ red $=M R$ $>2$ ) answer (for interpretation of the references to color in this figure legend, the reader is referred to the web version of this article).

\subsection{UV Systems}

UV irradiation showed, as also sand biofiltration did, a variety in OMPs behavior, with 3 categories on the ground: some compounds (e.g. diclofenac and estrogens) were remarkably eliminated (green category of Fig. 1), while others (erythromycin, carbamazepine and diazepam) fell in the orange category (i.e. low abatement). As extensively reported in [10], the reason lies in the different photosensitivity of target OMPs, whose removal efficiencies are very closely related to.

Moreover, it is well known [18] that UV treatment is not able to reach the complete mineralization of pollutants, but only to reduce their complexity owing to the rupture of bonds by UV light action. The transformation of parent compounds into by-products was clearly evidenced by the effect-based estrogenicity assay. Indeed, when we again linked the removal of the most critical pollutants exhibiting estrogenic activity (i.e., the 3 estrogens) with the reduced estrogenicity (Fig. 2), we recorded divergent outcomes: after the UV irradiation the real estrogenic burden measured with the bioassay remained almost unchanged in the effluent (only a $10 \%$ removal efficiency), despite the satisfactory abatement of the estrogens (> 80\%). As anticipated before, we can therefore attribute this outcome to the formation of transformation products that still possess estrogenic activity, but not detected by the target chemical analysis: for instance, the hydroxylation of estradiol into 2-hydroxyestradiol, which is able to form hydrogen bonds with the estrogen receptor, was observed by [18] after UV. This behavior, therefore, could explain the persistence of the estrogenic activity after UV treatment, as also observed in other works ([19] among others). On the contrary, it is highly unlikely that the low estrogenicity removal could be attributable to the low elimination rate of the UV technique recorded for other micropollutants (CBZ, DZP, etc).

As mutagenicity is concerned, UV system exhibited again some weaknesses (Fig. 4). While the determination of the mutagenicity was not possible in the influent at the highest tested doses (25and 50-mL wastewater equivalent/plate), because of the strong toxic 


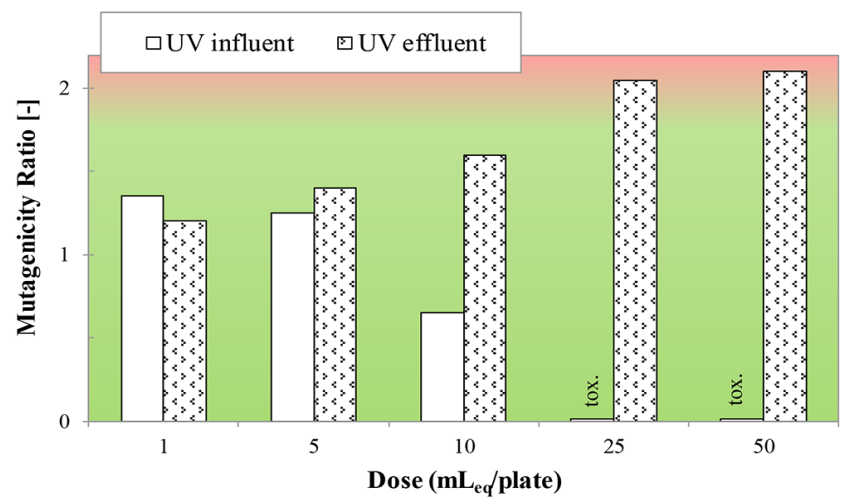

Fig. 4. Mutagenicity ratio obtained using Ames test, for UV treatment (tox $=$ toxic to bacteria). Background color indicates a negative (green $=M R<2)$ or positive $($ red $=M R>2$ ) answer (for interpretation of the references to color in this figure legend, the reader is referred to the web version of this article).

effect on bacteria, the effluent maintained a borderline mutagenicity strength, with $M R$ values slightly above the threshold of 2 . Similar findings were also achieved by other authors [20], that have shown how UV irradiation could lead to the formation of potential mutagenic by-products.

\section{Conclusions}

This study represents the last piece of a bigger "puzzle", preceded by the works [9] and [10] that analysed the behaviour of several organic micropollutants in three post-treatment technologies for secondary effluents polishing. In this last work, their efficiency was evaluated towards two specific modes of toxic actions (the most commonly studied in wastewater: estrogenic and mutagenic activity), with an integrated chemical-biological approach, i.e. by coupling the information derived from OMPs detection with those derived from the effect-based approach (estrogenic and mutagenic activity).

The results indicated that bioassays are needed to correctly gauge the ecohazard posed by micropollutants, as there was no direct link between OMPs removal and toxicity attenuation. In the case of estrogenicity, indeed, although both biofiltration and UV treatment satisfactory eliminated the estrogens (E1, E2, EE2), only the former was able to make the estrogenic load plummet, while UV treatment left it almost unchanged. Moreover, unlike biofilters, after UV treatment also a slight mutagenic effect was recorded. Therefore, effect-based assays suggested how photolysis could generate by-products, missed by the target chemical analysis but still bioactive and therefore detected by bioassays.

In conclusion, the application of a multitiered bio-chemical strategy may allow to properly profile tertiary treatments, and eventually leading to the optimization of their operational conditions in order to obtain a removal not only of OMPs, but also of their toxicity.

\section{Acknowledgment}

This work was conceived within a Short Term Scientific Mission
(STSM) of the Water2020 Cost Action ES1202: Conceiving Wastewater Treatment in 2020 | Energetic, environmental and economic challenges. Authors from Universidade de Santiago de Compostela belong to the Galician Competitive Research Group GRC 2013-032 and to the CRETUS Strategic Partnership (AGRUP2015/02). All these programmes are co-funded by FEDER (UE).

We thank dr. Elisabetta Ceretti and dr. Ilaria Zerbini for their precious collaboration in laboratory activities for mutagenicity studies. We thank Giuseppe Ruzzante for his editorial assistance.

This article was presented at IWA 11th Micropol \& Ecohazard Conference (2019) held on 20-24 October 2019 in Seoul, South Korea.

\section{Author Contributions}

M.P. (Research Fellow) and L.P. (Research Fellow) designed the experiments, analyzed data and co-wrote the paper; L.P. (Research Fellow) operate the reactors and carried out micropollutants detection; D.F. (Professor) and G.V. (Research Fellow) conducted the mutagenicity tests; G.M. (Professor) and N.S. (Research Fellow) conducted the estrogenicity tests; R.P. (Assistant Professor), J.L. (Professor), F.O. (Professor) and M.C. (Professor) supervised the research and critically reviewed the paper.

\section{References}

1. Luo Y, Guo W, Ngo HH, et al. A review on the occurrence of micropollutants in the aquatic environment and their fate and removal during wastewater treatment. Sci. Total Environ. 2014;473-474:619-641.

2. Escher BI, Allinson M, Altenburger R, et al. Benchmarking organic micropollutants in wastewater, recycled water and drinking water with in vitro bioassays. Environ. Sci. Technol. 2014;48:1940-1956.

3. Reungoat J, Escher BI, Macova M, Keller J. Biofiltration of wastewater treatment plant effluent: Effective removal of pharmaceuticals and personal care products and reduction of toxicity. Water Res. 2011;45:2751-2762.

4. Leusch FDL, Neale PA, Arnal C, et al. Analysis of endocrine activity in drinking water, surface water and treated wastewater from six countries. Water Res. 2018;139:10-18.

5. Lundqvist J, Mandava G, Lungu-Mitea S, Lai FY, Ahrens L. In vitro bioanalytical evaluation of removal efficiency for bioactive chemicals in Swedish wastewater treatment plants. Sci. Rep. 2019;9:1-9.

6. Välitalo P, Massei R, Heiskanen I, et al. Effect-based assessment of toxicity removal during wastewater treatment. Water Res. 2017;126:153-163.

7. Gonzalez-Gil L, Papa M, Feretti D, et al. Is anaerobic digestion effective for the removal of organic micropollutants and biological activities from sewage sludge? Water Res. 2016;102.

8. Ternes TA, Prasse C, Eversloh CL, et al. Integrated Evaluation Concept to Assess the Efficacy of Advanced Wastewater Treatment Processes for the Elimination of Micropollutants and 
Pathogens. Environ. Sci. Technol. 2017;51:308-319.

9. Paredes L, Fernandez-Fontaina E, Lema JM, Omil F, Carballa M. Understanding the fate of organic micropollutants in sand and granular activated carbon biofiltration systems. Sci. Total Environ. 2016;551-552:640-648.

10. Paredes L, Omil F, Lema JM, Carballa M. What happens with organic micropollutants during UV disinfection in WWTPs? A global perspective from laboratory to full-scale. J. Hazard. Mater. 2018;342:670-678.

11. Pei M, Zhang B, He Y, et al. State of the art of tertiary treatment technologies for controlling antibiotic resistance in wastewater treatment plants. Environ. Int. 2019;131:105026.

12. Escher BI, Aït-Aïssa S, Behnisch PA, et al. Effect-based trigger values for in vitro and in vivo bioassays performed on surface water extracts supporting the environmental quality standards (EQS) of the European Water Framework Directive. Sci. Total Environ. 2018;628-629:748-765.

13. Escher B, Leusch F. Bioanalytical tools in water quality assessment. London: IWA publishing; 2012.

14. APHA. Standard Methods for the Examination of Water and Wastewater 22nd ed. Washington DC: American Public Health Association; 2008.

15. Rao K, Li N, Ma M, Wang Z. In vitro agonistic and antagonistic endocrine disrupting effects of organic extracts from waste water of different treatment processes. Front. Environ. Sci. Eng. 2014;8:69-78.

16. Prasse C, Stalter D, Schulte-Oehlmann U, Oehlmann J, Ternes TA. Spoilt for choice: A critical review on the chemical and biological assessment of current wastewater treatment technologies. Water Res. 2015;87:237-270.

17. Hermes N, Jewell KS, Schulz M, et al. Elucidation of removal processes in sequential biofiltration (SBF) and soil aquifer treatment (SAT) by analysis of a broad range of trace organic chemicals (TOrCs) and their transformation products (TPs). Water Res. 2019;163:114857.

18. Mathon B, Choubert JM, Miege C, Coquery M. A review of the photodegradability and transformation products of 13 pharmaceuticals and pesticides relevant to sewage polishing treatment. Sci. Total Environ. 2016;551-552:712-724.

19. Zhang W, Li Y, Wu Q, Hu H. Removal of endocrine-disrupting compounds, estrogenic activity, and escherichia coliform from secondary effluents in a TiO 2-coated photocatalytic reactor. Environ. Eng. Sci. 2012;29:195-201.

20. Jia A, Escher BI, Leusch FDL, et al. In vitro bioassays to evaluate complex chemical mixtures in recycled water. Water Res. 2015;80:1-11. 